\title{
Impact of Powered and Tissue-Specific Endoscopic Stapling Technology on Clinical and Economic Outcomes of Video-Assisted Thoracic Surgery Lobectomy Procedures: A Retrospective, Observational Study
}

Daniel L. Miller · Sanjoy Roy · Edmund S. Kassis · Sashi Yadalam •

Sushama Ramisetti · Stephen S. Johnston

Received: January 18, 2018 / Published online: April 16, 2018

(C) The Author(s) 2018

\begin{abstract}
Introduction: Video-assisted thoracic surgery (VATS) lung resections are complex procedures with a critical role played by endoscopic staplers in the transection of vessels, bronchi, and lung tissue. This retrospective, observational study compared hospital resource use, costs, and complications of VATS lobectomy procedures for whom powered versus manual endoscopic surgical staplers were used.
\end{abstract}

Enhanced content To view enhanced content for this article go to https://doi.org/10.6084/m9.figshare. 5882767.

Electronic supplementary material The online version of this article (https://doi.org/10.1007/s12325$018-0679-z)$ contains supplementary material, which is available to authorized users.

D. L. Miller

WellStar Health System, Mayo Clinic Care Network, Marietta, GA, USA

S. Roy

Franchise Health Economics and Market Access,

Ethicon, Inc., Somerville, NJ, USA

E. S. Kassis

Global Medical Affairs, Ethicon, Cincinnati, OH, USA

S. Yadalam · S. Ramisetti · S. S. Johnston $(\bowtie)$

Epidemiology, Medical Devices, Johnson \&

Johnson, New Brunswick, NJ, USA

e-mail: sjohn147@its.jnj.com
Methods: Patients $\geq 18$ years of age undergoing elective VATS lobectomy during an inpatient admission from January 1, 2012 to September 30, 2016 were identified from the Premier Healthcare Database (first admission $=$ index admission). Use of either powered or manual endoscopic staplers during the index admission was identified from hospital administrative records. Multivariable regression analyses adjusting for patient, hospital, and provider characteristics and hospital-level clustering were carried out to compare the following outcomes between the powered and manual stapler groups: hospital length of stay (LOS), operating room time (ORT), hospital costs, complications (bleeding and/or transfusions, air leak complications, pneumonia, and infection), discharge status, and 30-, 60-, and 90-day allcause readmissions.

Results: The powered and manual stapler groups comprised 659 patients (mean age 66.1 years; $53.6 \%$ female) and 3100 patients (mean age 66.7 years; $54.8 \%$ female), respectively. In the multivariable analyses, the powered stapler group had shorter LOS (4.9 vs. 5.9 days, $P<0.001$ ), lower total hospital costs $(\$ 23,841$ vs. $\$ 26,052, P=0.009)$, and lower rates of combined hemostasis complications (bleeding and/or transfusions; $8.5 \%$ vs. $16.0 \%$, $P<0.001)$ and transfusions $(4.3 \%$ vs. $6.8 \%$, $P=0.002)$, compared with the manual stapler group. Other outcomes did not differ significantly between the study groups. Similar trends 
were observed in subanalyses comparing devices across predominant manufacturers in each group, and in subanalyses of patients with comorbid chronic obstructive pulmonary disease.

Conclusion: In this analysis of VATS lobectomy procedures, powered staplers were associated with significant benefits with respect to selected types of hospital resource use, costs, and clinical outcomes when compared with manual staplers.

Funding: Johnson \& Johnson.

Keywords: Complications; Hospital costs; Hospital resource use; Lobectomy; Manual stapler; Powered stapler; Video-assisted thoracic surgery

\section{INTRODUCTION}

The video-assisted thoracic surgery (VATS) approach has emerged as a superior option in comparison with open thoracotomy because of benefits to patients associated with the minimally invasive technique [1-7]. Studies have consistently shown that VATS lobectomy is associated with lower complication rates and morbidity when compared with open thoracotomy [1-4]. Additionally, recently published series of cancer patients showed that VATS lobectomy was associated with less postoperative pain, improved quality of life, and similar hospital costs when compared with open thoracotomy [5-7].

Owing to the constrained operative space, VATS lobectomy procedures are complex, especially in patients with cancer. Stapling is a critical step during VATS lobectomy procedures for the transection of pulmonary veins and arteries, bronchi, and lung parenchyma. Innovation in endoscopic stapling technology has introduced powered devices, which utilize a motor for both staple firing and knife blade action. In 2010, the US FDA approved the first powered stapler-the iDrive $^{\mathrm{TM}}$ powered stapling system-produced by Covidien (now Medtronic) [8]. In 2011, the US FDA approved the Echelon Flex ${ }^{\mathrm{TM}}$ powered endopath stapler, produced by Ethicon $[9,10]$. Subsequent versions of powered endoscopic stapling devices have been released by Medtronic and Ethicon [11, 12]. In 2015, Ethicon/ Johnson \& Johnson launched the Echelon Flex $^{\mathrm{TM}}$ powered vascular stapler (PVS), which is designed specifically for thoracic procedures to provide detailed precision in access to, placement around, firing, and division of the delicate pulmonary vessels [13].

These recent developments in stapling technology may have the potential to improve patient outcomes in these complex procedures. For example, a recently published observational study of patients who underwent laparoscopic Roux-en-Y gastric bypass or laparoscopic sleeve gastrectomy found that the use of powered staplers versus manual staplers was associated with lower total hospital costs and a lower rate of bleeding/transfusions [14].

Currently, there is a lack of similar such data for VATS lobectomy procedures, in which newer and more tissue-specific stapling technology may have a particularly important impact. Thus, the purpose of this retrospective, observational study was to compare hospital resource use, costs, and complications of VATS lobectomy procedures in US patients for whom powered or manual endoscopic surgical staplers were used.

\section{METHODS}

\section{Study Data Source and Patient Selection}

This study was undertaken from a US hospital perspective. The data source was the Premier Healthcare Database, which contains complete clinical coding, hospital cost, and patient billing data from more than 700 hospitals throughout the USA. Although the database excludes federally funded hospitals (e.g., Veterans Affairs), the hospitals included are nationally representative on the basis of bed size, geographic region, location (urban/rural), and teaching status. The database contains datestamped records of all billed items including medications; laboratory, diagnostic, and therapeutic services; and primary and secondary diagnoses for each patient's hospitalization. 
Additionally, the database also provides patient demographic and payer information.

Figure 1 shows the study's patient selection process. Patients selected for the study underwent VATS lobectomy, as defined by International Classification of Diseases, Ninth Revision, Clinical Modification (ICD-9-CM) procedure coding and International Classification of Diseases, Tenth Revision, Procedure Coding System (ICD-10-PCS) coding (See Supplemental Appendix 1 for a listing of all codes used in the study) during a hospital admission between January 1, 2012 and September 30, 2016. The first hospital admission for VATS lobectomy during this period was defined as the index admission, and patients were required to be at

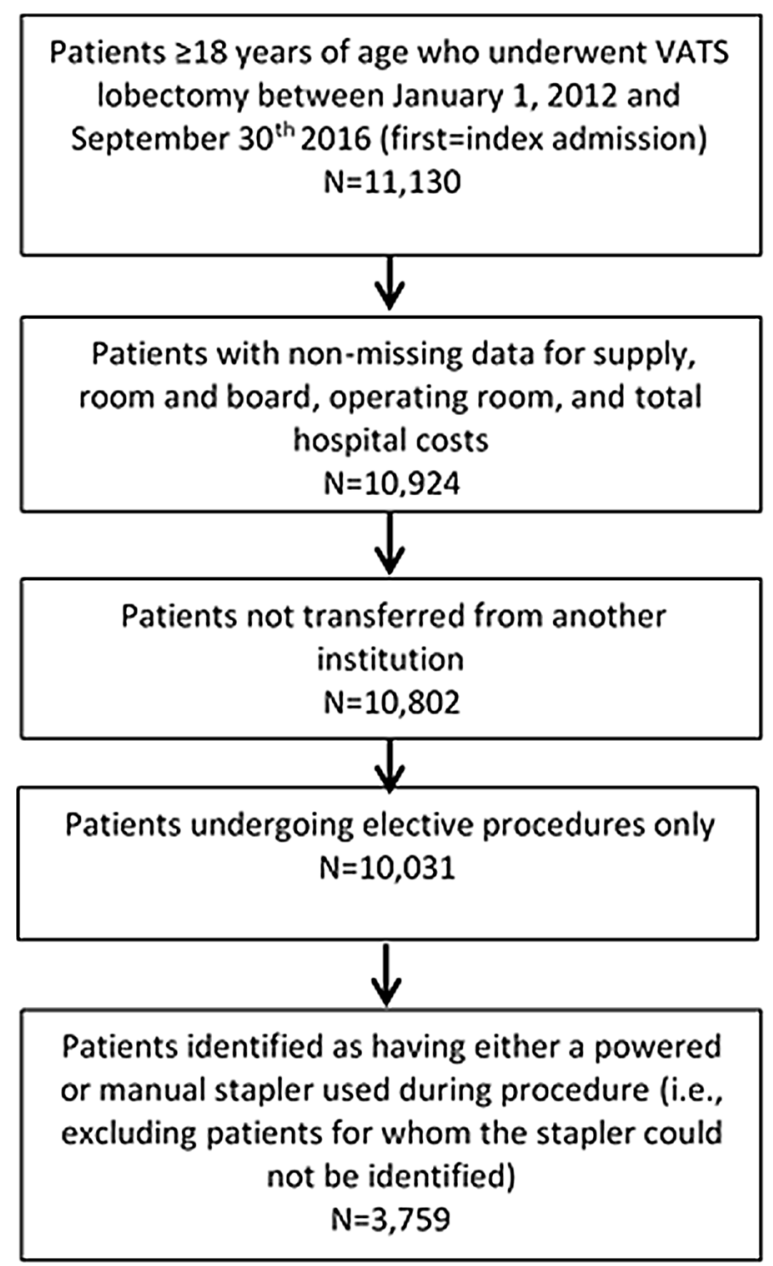

Fig. 1 Selection of study cohorts. VATS video-assisted thoracic surgery least 18 years of age at the time of the index admission. Patients were excluded from the study if they had missing data on hospital supply, room and board, or total hospital costs, if they were transferred from another institution, or if they had a non-elective VATS lobectomy.

Use of either powered or manual staplers during the index hospitalization was identified from hospital administrative records by searching for various combinations of device names (e.g., iDrive, Powered Echelon Flex, Powered Vascular Stapler), model numbers (e.g., PCE45A, IDRVULTRA1, PVE35A), and/or descriptors of devices being "powered". Staplers were also further classified by manufacturer (Ethicon/ Johnson \& Johnson; Medtronic/Covidien). Only patients for whom a stapler used during the index hospitalization could be identified as either powered or manual were retained for the study; patients with evidence of both powered and manual staplers were excluded from the study because of the inability to assign them to one of the two study groups: powered stapler group or manual stapler group. Patients were also excluded if da Vinci EndoWrist ${ }^{\circledR}$ surgical staplers (Intuitive Surgical, Sunnyvale, CA), which are part of the robotic da Vinci Surgical System, were used during the index hospitalization.

\section{Patient and Hospital/Provider Characteristics}

Patient demographics and hospital/provider characteristics measured during the index admission included age, gender, marital status, race, Hispanic vs. non-Hispanic indicator, payer type, urban or rural hospital, hospital teaching status, hospital geographic region, hospital bed size, procedural physician specialty, year of surgery/index admission, hospital surgical volume for VATS lobectomy, and an indicator for whether hospital costs are derived from a costto-charge ratio or procedural costing.

Patient clinical characteristics measured during the index admission included the Charlson comorbidity index (CCI) [15], the day on which the VATS lobectomy procedure was performed after hospital admission, cancer vs. 
non-cancer primary diagnosis, concomitant wedge resection, concomitant segmentectomy resection, robotic assistance, and several individual comorbidities from the Charlson and Elixhauser comorbidity indices [based on diagnoses recorded in any position: cancer (metastatic), cancer (non metastatic), cerebrovascular disease, chronic pulmonary disease, coagulopathy, congestive heart failure, connective tissue/ rheumatic disease, depression, diabetes (w/complications), diabetes (w/o complications), hypertension (complicated), hypertension (uncomplicated), hypothyroidism, liver disease (mild), myocardial infarction, neurological disorders, obesity, peripheral vascular disease, renal disease, valvular disease, and weight loss] [15]. Comorbidities and the CCI were measured through the presence of ICD-9$\mathrm{CM} / \mathrm{ICD}-10-\mathrm{CM}$ codes, excluding those for which there was an indication that the comorbidity was not present on admission. Certain comorbidities from the Charlson and Elixhauser comorbidity indices were excluded because of infrequency or non-occurrence (e.g., paralysis, human immunodeficiency virus/acquired immunodeficiency deficiency syndrome).

\section{Outcomes}

Economic and healthcare resource use outcomes were evaluated during the index admission (with the exception of readmissions) and included hospital length of stay (LOS); total hospital costs from the hospital perspective (including subcategories of medical/surgical supply costs, room and board costs, and operating room costs); operating room time; discharge to a non-home setting (e.g., skilled nursing facility, intermediate care facility) vs. discharge to home with or without home healthcare; and 30-, 60-, and 90-day all-cause readmissions to the hospital in which the VATS lobectomy procedure was performed. All costs were inflation adjusted to 2016 US dollars using the Medical Care component of the US Bureau of Labor Statistics Consumer Price Index. When we analyzed the operating room time outcome, patients were included only if their operating room time values recorded in the database fell between $30 \mathrm{~min}$ and $24 \mathrm{~h}(86.5 \%$ of patients in the primary analysis met this criterion); this step was taken to eliminate patients with implausible values. When we analyzed the allcause readmission outcomes, patients were included only if the hospital in which their VATS lobectomy procedure was performed had (at an overall hospital level) discharge records extending throughout the observation period of interest $(30,60$, or 90 days); this step was taken to eliminate patients for whom all-cause readmission would be unobservable because of noncontribution of discharge data at a hospital level.

Clinical outcomes were evaluated during the index admission and included a composite outcome of bleeding and/or transfusions; transfusions alone; acute posthemorrhagic anemia; air leak complications (based on the ICD-9-CM/ICD-10-CM diagnosis codes for pneumothorax or air leak, which includes but is not necessarily limited specifically to prolonged air leaks); pneumonia; and infection (comprising surgical site, septicemia, pneumonia, and infections of other sites [see Supplemental Appendix 1]).

Within the study protocol, the operating room time, all-cause readmission, and clinical complication outcomes were designated as exploratory, while other outcomes were designated as primary. The exploratory outcomes were designated as such because of (1) uncertainty regarding the accuracy of values recorded for operating room time; (2) readmissions to the hospital within the Premier Healthcare Database are captured only when the patient returns to the hospital in which the index admission took place, thereby introducing the potential for incomplete outcome data capture; and (3) uncertainty regarding the sensitivity and specificity of the clinical coding for complications.

\section{Statistical Analyses}

Bivariate analyses, stratified by the powered vs. manual stapler groups, were used to describe patient and hospital/provider characteristics and unadjusted outcomes. Standardized differences were used to assess the magnitude of 
differences in baseline characteristics between the study groups, where a standardized difference greater than 0.10 was considered to be imbalanced.

Multivariable regression models were used to compare outcomes between the powered and the manual stapler group, adjusting for all aforementioned patient and hospital/provider characteristics, regardless of the values of the standardized differences between study groups. The Box Cox and modified Park test were used, respectively, to select link functions and error distributions which were tailored to the empirical distributions of the outcome variables (e.g., log link and gamma error distribution for hospital costs; logit link and binomial error distribution for dichotomous outcomes) [16, 17]. Statistical clustering may arise among patients who receive treatment within the same hospital; thus, this was addressed using generalized estimating equations (GEE) models and mixed models. The former used an exchangeable working correlation structure-chosen on the basis of a qualitative understanding of the potential nature of clustering within hospitals. Mixed models were used when GEE models failed to converge, which was primarily the case for dichotomous outcomes. In the GEE models, inference was based on empirical (robust) standard error estimates. Adjusted outcomes were generated for each of the comparator groups using the recycled prediction (marginal standardization approach) [18]. A two-sided critical value of 0.05 was used to determine statistical significance. All statistical analyses were performed using SAS version 9.3.

\section{Subgroup Analyses}

First, to test whether the powered or manual findings were driven by manufacturer-level effects, post hoc subgroup analyses were completed wherein the predominant manufacturers within the powered and manual stapler groups were compared to one another for all study outcomes using the same statistical analysis approach as the primary analyses; specifically, Ethicon powered staplers, which accounted for $99.4 \%$ of the powered staplers, and Medtronic manual staplers, which accounted for $75.8 \%$ of the manual staplers.

Second, to examine the role of the thoracic vessel tissue-specific design of the Ethicon PVS stapler, post hoc subgroup analyses were completed wherein the PVS stapler was compared to Medtronic manual staplers for all study outcomes using the same statistical analysis approach as the primary analyses. These analyses are restricted to only the years in which the PVS stapler was present in the database (2015 and 2016). Furthermore, to increase the likelihood that the Ethicon PVS stapler was used for vascular transections, evidence of a second nonPVS Ethicon powered stapler being used during the VATS lobectomy procedure was required for the Ethicon PVS stapler group.

Finally, the prevalence of comorbid chronic obstructive pulmonary disease (COPD) is relatively high among patients undergoing lung lobectomies, and has been associated with perioperative morbidity and mortality [19]. Thus, analyses of hemostasis-related complications were repeated in the subgroup of patients with a comorbid diagnosis of chronic obstructive pulmonary disease (codified as chronic obstructive pulmonary disease and allied disorders, according to the ICD-9-CM/ICD-10-CM taxonomies) for the primary analysis and the post hoc subgroup analysis comparing outcomes between Ethicon powered staplers and Medtronic manual staplers.

\section{Propensity Score Matched Sensitivity Analysis}

Finally, a sensitivity analysis was conducted to determine whether the study's primary findings are robust to use of an alternative statistical approach: propensity score matching. Specifically, the powered stapler group was propensity score matched to the manual stapler group using a variable ratio matching approach of up to three manual stapler patients per one powered stapler patient. The propensity score was estimated in multivariable logistic regression using all covariates from the main multivariable models as predictors of membership in the powered stapler cohort (vs. manual stapler 
cohort as reference). The matching was accomplished through greedy matching, with a caliper equal to 0.2 times the standard deviation of the logit of the propensity score. After matching, the post-match balance of covariates was examined via standardized differences, and variables which remained imbalanced (standardized difference $>0.10$ ) across the propensity matched cohorts were entered into a second-stage multivariable outcome model in which the primary predictor was membership in the powered stapler cohort (vs. manual stapler cohort as reference). The second-stage multivariable outcome models were implemented in the same manner as the primary analysis models and adjusted outcomes were generated for each of the comparator groups using the recycled predication. As a result of the comparatively smaller sample sizes of the subgroup analyses, which after matching would have likely yielded inadequate statistical power, this sensitivity analysis was conducted only for the primary analysis.

\section{Study Conduct and Protection of Human Subjects}

This study was conducted per a prespecified protocol which was approved through scientific governance. As a result of the retrospective, observational nature of the study, it was not a registered trial. The study was planned and conducted in a manner consistent with the International Society of Pharmacoepidemiology principles of the Good Research for Comparative Effectiveness guidance and the PICO Model for Clinical Questions [20, 21]. The Premier Healthcare Database consists of de-identified healthcare records. In the USA, retrospective analyses of the Premier Healthcare Database data are considered exempt from institutional review board (IRB) oversight as dictated by Title 45 Code of Federal Regulations, Part 46 of the USA, specifically 45 CFR 46.101(b)(4) (http:// www.hhs.gov/ohrp/humansubjects/guidance/ $45 \mathrm{cfr} 46 . \mathrm{html})$. In addition, in accordance with the HIPAA Privacy Rule, disclosed data from the Premier Healthcare Database are considered de-identified per 45 CFR 164.506(d)(2)(ii)(B) through the "Expert determination" method (http://www.hhs.gov/hipaa/for-professionals/pri vacy/special-topics/de-identification/). Throughout this research project, the study data remained de-identified and stored on encrypted, passwordprotected servers to protect patient confidentiality. This article does not contain any studies with human participants or animals performed by any of the authors.

\section{RESULTS}

\section{Patient Demographics, Clinical Characteristics, and Hospital/Provider Characteristics}

Patient demographics, clinical characteristics, and hospital/provider characteristics are shown in Tables 1, 2, and 3, respectively. A total of 659 patients were identified as having undergone VATS lobectomy with powered endoscopic staplers $(99.4 \%$ Ethicon manufactured; 0.6\% Medtronic manufactured) and 3100 with manual staplers used $(75.8 \%$ Medtronic manufactured and 26.7\% Ethicon manufactured; $2.6 \%$ with both).

The powered and manual stapler groups had a mean age of 66.1 and 66.7 years and $53.6 \%$ and $54.8 \%$ were female, respectively. Medicare was the most common payer type, accounting for $62 \%$ of each study group. On the basis of the standardized difference, the powered and manual stapler groups were similar in age, gender, and payer types, but differed in marital status, race, Hispanic ethnicity, and index hospitalization year (Table 1). The groups had similar prevalence rates of almost all comorbidities examined, except for chronic obstructive pulmonary disease $(44.1 \%$ manual, $49.2 \%$ powered) and (diagnosed) obesity (10.5\% manual, $14.3 \%$ powered). The groups differed in the proportion of patients with a primary discharge diagnosis of cancer $(91.2 \%$ powered stapler group; 94.6\% manual stapler group) and in the proportion of patients for whom there was robotic assistance during the procedure $(31.1 \%$ powered stapler group; $9.2 \%$ manual stapler group) (Table 2). The groups differed 
Table 1 Patient demographics, primary analyses

\begin{tabular}{|c|c|c|c|}
\hline & $\begin{array}{l}\text { Powered } \\
\text { staplers, } \\
n=659\end{array}$ & $\begin{array}{l}\text { Manual } \\
\text { staplers, } \\
n=3100\end{array}$ & $\begin{array}{l}\text { Std. } \\
\text { diff. }^{\text {a }}\end{array}$ \\
\hline Age, mean (SD) & $66.1(10.7)$ & $66.7(10.2)$ & 0.059 \\
\hline \multicolumn{4}{|l|}{ Age group, $n(\%)$} \\
\hline $18-44$ & $23(3.5)$ & $65(2.1)$ & 0.085 \\
\hline $45-54$ & $54(8.2)$ & $309(10.0)$ & 0.062 \\
\hline $55-64$ & $171(25.9)$ & $794(25.6)$ & 0.008 \\
\hline$\geq 65$ & $411(62.4)$ & $1932(62.3)$ & 0.001 \\
\hline \multicolumn{4}{|l|}{ Sex, $n(\%)$} \\
\hline Female & $353(53.6)$ & $1699(54.8)$ & 0.025 \\
\hline Male & $306(46.4)$ & $1401(45.2)$ & \\
\hline \multicolumn{4}{|c|}{ Marital status, $n(\%)$} \\
\hline Married & $397(60.2)$ & $1689(54.5)$ & 0.117 \\
\hline Single & $251(38.1)$ & $1193(38.5)$ & 0.008 \\
\hline Other & $11(1.7)$ & $218(7.0)$ & 0.265 \\
\hline \multicolumn{4}{|l|}{ Race, $n(\%)$} \\
\hline White & $571(86.6)$ & $2487(80.2)$ & 0.173 \\
\hline Black & $51(7.7)$ & $230(7.4)$ & 0.012 \\
\hline Other & $37(5.6)$ & $383(12.4)$ & 0.237 \\
\hline Hispanic, $n(\%)$ & $93(14.1)$ & $114(3.7)$ & 0.373 \\
\hline \multicolumn{4}{|l|}{ Payer type, $n(\%)$} \\
\hline Medicare & $405(61.5)$ & $1921(62.0)$ & 0.011 \\
\hline Commercial & $195(29.6)$ & $883(28.5)$ & 0.024 \\
\hline Medicaid & $34(5.2)$ & $165(5.3)$ & 0.007 \\
\hline Other & $25(3.8)$ & $131(4.2)$ & 0.022 \\
\hline \multicolumn{4}{|c|}{ Index hospitalization year } \\
\hline 2012 & $14(2.1)$ & $823(26.5)$ & 0.743 \\
\hline 2013 & $86(13.1)$ & 739 (23.8) & 0.281 \\
\hline 2014 & $145(22.0)$ & $614(19.8)$ & 0.054 \\
\hline 2015 & $245(37.2)$ & $591(19.1)$ & 0.411 \\
\hline 2016 & $169(25.6)$ & $333(10.7)$ & 0.393 \\
\hline
\end{tabular}

$S D$ standard deviation, Std. diff. standardized difference

a Standardized difference $>0.10$ indicates imbalance across the cohorts substantially on nearly all hospital and provider characteristics, including US geographic region, rural locale, cost type, surgical specialty, and surgical volume (Table 3 ).

\section{Multivariable-Adjusted Outcomes}

Unadjusted outcomes are available in Supplemental Appendix 2. Multivariable-adjusted outcomes for the primary analyses are shown in Table 4. After adjustment for patient demographics, clinical characteristics, and hospital/ provider characteristics, the powered stapler group had statistically significant shorter adjusted hospital LOS (4.87 vs. $5.88, P<0.001$ ); lower adjusted total hospital costs $(\$ 23,841$ vs. $\$ 26,052, P=0.009)$, and adjusted room and board costs ( $\$ 6737$ vs. $\$ 7945, P=0.001)$; and lower adjusted rates of the composite hemostasis complication outcome (bleeding and/or transfusion) ( $8.5 \%$ vs. $16.0 \%, P<0.001)$, and transfusion $(5.4 \%$ vs. $10.9 \%, P=0.002)$ compared with the manual stapler group. Differences between the study groups in supply costs, operating room costs, discharge to a nonhome setting, operating room time, acute posthemorrhagic anemia rates, air leak complication rates, pneumonia rates, infection rates, and all-cause readmission rates at 30, 60, and 90 days were not statistically significant.

\section{Subgroup Analyses}

Multivariable-adjusted outcomes for the post hoc subgroup analyses are shown in Tables 4 (bottom panel), 5, and 6. In the subanalysis comparing outcomes between the Ethicon powered and Medtronic manual stapler groups (Table 5), the Ethicon powered stapler group had statistically significant shorter adjusted hospital LOS (4.89 vs. 5.66, $P<0.037$ ); lower adjusted total hospital costs $(\$ 23,785$ vs. $\$ 26,180, \quad P=0.008)$, adjusted supply costs ( $\$ 5021$ vs. $\$ 5989, P=0.016)$, and adjusted room and board costs ( $\$ 6792$ vs. $\$ 7984, P=0.039$ ); and lower adjusted rates of the composite hemostasis complication outcome (bleeding and/or transfusion) $(8.2 \%$ vs. $13.9 \%, P=0.022)$, and transfusion $(4.7 \%$ vs. $9.3 \%, P=0.018)$ 
Table 2 Patient clinical characteristics, primary analyses

\begin{tabular}{|c|c|c|c|}
\hline & $\begin{array}{l}\text { Powered } \\
\text { staplers, } \\
n=659\end{array}$ & $\begin{array}{l}\text { Manual } \\
\text { staplers, } \\
n=3100\end{array}$ & $\begin{array}{l}\text { Std. } \\
\text { diff. }^{\text {a }}\end{array}$ \\
\hline $\begin{array}{l}\text { Primary diagnosis of } \\
\text { cancer, } n(\%)\end{array}$ & $601(91.2)$ & $2934(94.6)$ & 0.135 \\
\hline \multicolumn{4}{|l|}{ Comorbidities, $n(\%)$} \\
\hline $\begin{array}{l}\text { Cancer } \\
\text { (metastatic) }\end{array}$ & $96(14.6)$ & $418(13.5)$ & 0.031 \\
\hline $\begin{array}{l}\text { Cerebrovascular } \\
\text { disease }\end{array}$ & $16(2.4)$ & $82(2.7)$ & 0.014 \\
\hline $\mathrm{COPD}^{\mathrm{b}}$ & $324(49.2)$ & $1368(44.1)$ & 0.101 \\
\hline Coagulopathy & $13(2.0)$ & $53(1.7)$ & 0.020 \\
\hline $\begin{array}{l}\text { Congestive heart } \\
\text { failure }\end{array}$ & $33(5.0)$ & $138(4.5)$ & 0.026 \\
\hline $\begin{array}{l}\text { Connective tissue/ } \\
\text { rheumatic disease }\end{array}$ & $23(3.5)$ & $108(3.5)$ & 0.000 \\
\hline Depression & $76(11.5)$ & $389(12.6)$ & 0.031 \\
\hline $\begin{array}{l}\text { Diabetes } \\
\text { (w/complications) }\end{array}$ & $8(1.2)$ & $49(1.6)$ & 0.031 \\
\hline $\begin{array}{l}\text { Diabetes (w/o } \\
\text { complications) }\end{array}$ & $110(16.7)$ & $547(17.7)$ & 0.025 \\
\hline $\begin{array}{l}\text { Hypertension } \\
\text { (complicated) }\end{array}$ & $44(6.7)$ & $189(6.1)$ & 0.024 \\
\hline $\begin{array}{l}\text { Hypertension } \\
\text { (uncomplicated) }\end{array}$ & $392(59.5)$ & $1723(55.6)$ & 0.079 \\
\hline Hypothyroidism & $80(12.1)$ & $395(12.7)$ & 0.018 \\
\hline $\begin{array}{l}\text { Liver disease } \\
\text { (mild) }\end{array}$ & $15(2.3)$ & $60(1.9)$ & 0.024 \\
\hline $\begin{array}{l}\text { Myocardial } \\
\text { infarction }\end{array}$ & $40(6.1)$ & $189(6.1)$ & 0.001 \\
\hline $\begin{array}{l}\text { Neurological } \\
\text { disorders }\end{array}$ & $16(2.4)$ & $64(2.1)$ & 0.025 \\
\hline Obesity $^{c}$ & $94(14.3)$ & $324(10.5)$ & 0.116 \\
\hline $\begin{array}{l}\text { Peripheral vascular } \\
\text { disease }\end{array}$ & $56(8.5)$ & $275(8.9)$ & 0.013 \\
\hline Renal disease & $45(6.8)$ & $208(6.7)$ & 0.005 \\
\hline Valvular disease & $27(4.1)$ & $133(4.3)$ & 0.010 \\
\hline
\end{tabular}

Table 2 continued

\begin{tabular}{|c|c|c|c|}
\hline & $\begin{array}{l}\text { Powered } \\
\text { staplers, } \\
n=659\end{array}$ & $\begin{array}{l}\text { Manual } \\
\text { staplers, } \\
n=3100\end{array}$ & $\begin{array}{l}\text { Std. } \\
\text { diff. }^{\text {a }}\end{array}$ \\
\hline Weight loss & $12(1.8)$ & $64(2.1)$ & 0.018 \\
\hline \multicolumn{4}{|l|}{ CCI, $n(\%)$} \\
\hline $0-1$ & $70(10.6)$ & $247(8.0)$ & 0.091 \\
\hline 2 & $153(23.2)$ & $872(28.1)$ & 0.113 \\
\hline 3 & $189(28.7)$ & $908(29.3)$ & 0.013 \\
\hline 4 & $89(13.5)$ & $365(11.8)$ & 0.052 \\
\hline $5+$ & $158(24.0)$ & $708(22.8)$ & 0.027 \\
\hline $\begin{array}{l}\text { Day of procedure }{ }^{\mathrm{d}}, \\
\text { mean }(\mathrm{SD})\end{array}$ & $1.0(0.2)$ & $1.1(0.6)$ & 0.134 \\
\hline $\begin{array}{l}\text { Concomitant wedge } \\
\text { resection, } n(\%)\end{array}$ & $57(8.6)$ & $265(8.5)$ & 0.004 \\
\hline $\begin{array}{l}\text { Concomitant } \\
\text { segmentectomy, } \\
n(\%)\end{array}$ & $4(0.6)$ & $13(0.4)$ & 0.026 \\
\hline
\end{tabular}

$\begin{array}{llll}\text { Robot-assisted } & 205(31.1) & 284(9.2) & 0.569\end{array}$ procedure, $n(\%)$

CCI Charlson comorbidity index, COPD chronic obstructive pulmonary disease, $S D$ standard deviation, Std. diff. standardized difference

a Standardized difference $>0.10$ indicates imbalance across the cohorts

b Comprising chronic obstructive pulmonary disease and allied disorders, according to the ICD-9-CM/ICD-10-CM taxonomies

c Based on ICD-9-CM/ICD-10-CM coding generally corresponding to body mass index $\geq 30 \mathrm{~kg} / \mathrm{m}^{2}$

d Day on which VATS lobectomy procedure was performed; value of 1 indicates day of admission

compared with the Medtronic manual stapler group. Differences between the study groups in all other outcomes were not statistically significant.

In the subanalysis comparing outcomes between a combination of Ethicon PVS (which presumably was used for vascular transections 
Table 3 Hospital and provider characteristics, primary analyses

\begin{tabular}{|c|c|c|c|}
\hline & Powered staplers, $n=659$ & Manual staplers, $n=3100$ & Std. diff. ${ }^{a}$ \\
\hline \multicolumn{4}{|l|}{ US geographic region, $n(\%)$} \\
\hline South & $557(84.5)$ & $1445(46.6)$ & 0.870 \\
\hline Midwest & $4(0.6)$ & $174(5.6)$ & 0.291 \\
\hline Northeast & $16(2.4)$ & $999(32.2)$ & 0.856 \\
\hline West & $82(12.4)$ & $482(15.5)$ & 0.090 \\
\hline Rural, $n(\%)$ & $92(14.0)$ & $172(5.5)$ & 0.286 \\
\hline Non-teaching, $n(\%)$ & $297(45.1)$ & $1468(47.4)$ & 0.046 \\
\hline Cost type: cost-to-charge ratio ${ }^{\mathrm{b}}, n(\%)$ & $58(8.8)$ & $756(24.4)$ & 0.428 \\
\hline \multicolumn{4}{|l|}{ Bed Size } \\
\hline $0-299$ & $108(16.4)$ & $558(18.0)$ & 0.043 \\
\hline $300-399$ & $87(13.2)$ & $467(15.1)$ & 0.053 \\
\hline $400-499$ & $124(18.8)$ & $670(21.6)$ & 0.070 \\
\hline$\geq 500$ & $340(51.6)$ & $1405(45.3)$ & 0.126 \\
\hline \multicolumn{4}{|l|}{ Surgical specialty, $n(\%)$} \\
\hline Thoracic & $542(82.2)$ & $1869(60.3)$ & 0.500 \\
\hline Cardiovascular & $75(11.4)$ & $791(25.5)$ & 0.370 \\
\hline General & $6(0.9)$ & $246(7.9)$ & 0.347 \\
\hline Other & $36(5.5)$ & $194(6.3)$ & 0.034 \\
\hline \multicolumn{4}{|l|}{ Monthly surgical volume ratio ${ }^{c}, n(\%)$} \\
\hline$\leq 0.75$ & 77 (11.7) & $765(24.7)$ & 0.342 \\
\hline$\geq 0.75<2$ & $106(16.1)$ & $955(30.8)$ & 0.353 \\
\hline$\geq 2<4$ & $225(34.1)$ & $1082(34.9)$ & 0.016 \\
\hline$\geq 4$ & $251(38.1)$ & $298(9.6)$ & 0.708 \\
\hline
\end{tabular}

$S D$ standard deviation, Std. diff. standardized difference

${ }^{a}$ Standardized difference $>0.10$ indicates imbalance across the cohorts

${ }^{b}$ Procedural costing is reference

c Ratio of the number of VATS lobectomy procedures to the number of months the patient's hospital was present in database during the study period

but was not verifiable in the database) plus another Ethicon powered stapler (presumably for other lung tissue) and the Medtronic manual stapler group for the years during which the Ethicon PVS device was present in the database (2015 and 2016; Table 6), the Ethicon PVS stapler group had statistically significant shorter adjusted hospital LOS (4.99 vs. 5.82, $P=0.047$ ); and lower adjusted rates of the composite hemostasis complication outcome (bleeding and/or transfusion) ( $4.8 \%$ vs. $14.2 \%, P=0.010)$ compared with the Medtronic manual stapler group. Differences between the study groups in the transfusion outcome were also statistically 
Table 4 Multivariable-adjusted outcomes, primary analyses

\begin{tabular}{|c|c|c|c|c|}
\hline \multirow[t]{2}{*}{ Outcome } & \multicolumn{2}{|l|}{ Adjusted outcomes $^{a}$} & \multirow[t]{2}{*}{$P$} & \multirow{2}{*}{$\begin{array}{l}\text { \% difference } \\
\text { (powered - manual) } \\
(\%)\end{array}$} \\
\hline & $\begin{array}{l}\text { Powered staplers, } \\
n=659\end{array}$ & $\begin{array}{l}\text { Manual staplers, } \\
n=3100\end{array}$ & & \\
\hline Hospital LOS & 4.87 & 5.88 & $<0.001$ & -17.1 \\
\hline Total hospital costs & $\$ 23,841$ & $\$ 26,052$ & 0.009 & -8.5 \\
\hline Supply cost & $\$ 5234$ & $\$ 5672$ & 0.405 & -7.7 \\
\hline Room and board cost & $\$ 6737$ & $\$ 7945$ & 0.001 & -15.2 \\
\hline Operating room cost & $\$ 7095$ & $\$ 7187$ & 0.735 & -1.3 \\
\hline Discharge to non-home ${ }^{\mathrm{b}}$ & $8.2 \%$ & $7.7 \%$ & 0.762 & 6.6 \\
\hline Operating room time ${ }^{c}$ & 284 & 301 & 0.073 & -5.8 \\
\hline \multicolumn{5}{|l|}{ In-hospital complications } \\
\hline Bleeding/transfusion & $8.5 \%$ & $16.0 \%$ & $<0.001$ & -46.8 \\
\hline Transfusion & $5.4 \%$ & $10.9 \%$ & 0.002 & -50.6 \\
\hline APHA & $4.3 \%$ & $6.8 \%$ & 0.097 & -36.8 \\
\hline Air leak complications ${ }^{\mathrm{d}}$ & $22.6 \%$ & $25.3 \%$ & 0.324 & -10.5 \\
\hline Pneumonia & $4.1 \%$ & $3.5 \%$ & 0.569 & 19.4 \\
\hline Infection & $4.4 \%$ & $3.8 \%$ & 0.612 & 16.5 \\
\hline \multicolumn{5}{|l|}{ All-cause readmission $^{\mathrm{e}}$} \\
\hline$\leq 30$ days & $3.1 \%$ & $4.4 \%$ & 0.217 & -30.5 \\
\hline$\leq 60$ days & $5.3 \%$ & $7.3 \%$ & 0.159 & -27.1 \\
\hline$\leq 90$ days & $7.5 \%$ & $9.8 \%$ & 0.171 & -23.5 \\
\hline \multicolumn{5}{|c|}{ Chronic obstructive pulmonary disease subgroup analyses ${ }^{f}$} \\
\hline Bleeding/transfusion & $8.6 \%$ & $18.9 \%$ & 0.002 & $-54.4 \%$ \\
\hline Transfusion & $6.2 \%$ & $13.2 \%$ & 0.011 & $-53.2 \%$ \\
\hline APHA & $4.1 \%$ & $7.9 \%$ & 0.113 & $-48.2 \%$ \\
\hline
\end{tabular}

APHA acute posthemorrhagic anemia, LOS length of stay

a Recycled prediction method; The following outcome models had questionable fit due to estimated G matrix not positive definite: all-cause readmission at 30,60, and 90 days

b Discharge to a non-home setting (e.g., skilled nursing facility, intermediate care facility) vs. discharge to home with or without home healthcare

c In minutes; $n$ with operating room time data $=542$ powered; 2711 manual; operating room time values trimmed to fall between $30 \mathrm{~min}$ and $24 \mathrm{~h}$

d Composite of air leak and/or pneumothorax

e 30-, 60-, and 90-day all-cause readmissions to the hospital in which the VATS lobectomy procedure was performed; $N$ with hospital-level follow-up data for at least 30 days $=624$ powered; 3018 manual; for at least 60 days $=581$ powered; 2942 manual; for at least 90 days $=545$ powered; 2866 manual

f Comprising chronic obstructive pulmonary disease and allied disorders, according to the ICD-9-CM/ICD-10-CM taxonomies; $n=324$ powered; 1368 manual 
Table 5 Multivariable-adjusted outcomes, Ethicon powered and Medtronic manual post hoc subanalysis

\begin{tabular}{|c|c|c|c|c|}
\hline \multirow[t]{2}{*}{ Outcome } & \multicolumn{2}{|c|}{ Adjusted outcomes ${ }^{a}$} & \multirow[t]{2}{*}{$P$} & \multirow{2}{*}{$\begin{array}{l}\% \text { difference (Ethicon } \\
\text { powered - Medtronic } \\
\text { manual) (\%) }\end{array}$} \\
\hline & $\begin{array}{l}\text { Ethicon powered } \\
\text { staplers, } n=655\end{array}$ & $\begin{array}{l}\text { Medtronic manual } \\
\text { staplers, } n=2351\end{array}$ & & \\
\hline Hospital LOS & 4.89 & 5.66 & 0.037 & -13.6 \\
\hline Total hospital costs & $\$ 23,785$ & $\$ 26,180$ & 0.008 & -9.1 \\
\hline Supply cost & $\$ 5021$ & $\$ 5989$ & 0.016 & -16.2 \\
\hline Room and board cost & $\$ 6792$ & $\$ 7984$ & 0.039 & -14.9 \\
\hline Operating room cost & $\$ 7032$ & $\$ 7225$ & 0.646 & -2.7 \\
\hline Discharge to non-home ${ }^{b}$ & $9.0 \%$ & $7.6 \%$ & 0.476 & 17.4 \\
\hline Operating room time $e^{c}$ & 278 & 302 & 0.090 & -7.9 \\
\hline \multicolumn{5}{|l|}{ In-hospital complications } \\
\hline Bleeding/transfusion & $8.2 \%$ & $13.9 \%$ & 0.022 & -40.7 \\
\hline Transfusion & $4.7 \%$ & $9.3 \%$ & 0.018 & -49.8 \\
\hline APHA & $4.9 \%$ & $6.1 \%$ & 0.469 & -20.2 \\
\hline Air leak complications ${ }^{\mathrm{d}}$ & $24.3 \%$ & $24.2 \%$ & 0.972 & 0.5 \\
\hline Pneumonia & $3.9 \%$ & $3.6 \%$ & 0.782 & 9.8 \\
\hline Infection & $4.5 \%$ & $4.0 \%$ & 0.689 & 13.6 \\
\hline \multicolumn{5}{|l|}{ All-cause readmission $^{\mathrm{e}}$} \\
\hline$\leq 30$ days & $3.0 \%$ & $4.2 \%$ & 0.317 & -28.0 \\
\hline$\leq 60$ days & $5.2 \%$ & $7.3 \%$ & 0.176 & -28.4 \\
\hline$\leq 90$ days & $7.4 \%$ & $9.8 \%$ & 0.187 & -24.8 \\
\hline \multicolumn{5}{|c|}{ Chronic obstructive pulmonary disease subgroup analyses ${ }^{f}$} \\
\hline Bleeding/transfusion & $8.0 \%$ & $15.9 \%$ & 0.025 & -49.8 \\
\hline Transfusion & $5.4 \%$ & $11.2 \%$ & 0.044 & -51.9 \\
\hline APHA & $3.7 \%$ & $7.2 \%$ & 0.177 & -48.3 \\
\hline
\end{tabular}

APHA acute posthemorrhagic anemia, LOS length of stay

a Recycled prediction method; The following outcome models had questionable fit due to estimated G matrix not positive definite: all-cause readmission at 30,60, and 90 days; pneumonia; infection

b Discharge to a non-home setting (e.g., skilled nursing facility, intermediate care facility) vs. discharge to home with or without home healthcare

${ }^{c}$ In minutes; $n$ with operating room time data $=538$ powered; 2190 manual; operating room time values trimmed to fall between $30 \mathrm{~min}$ and $24 \mathrm{~h}$

d Composite of air leak and/or pneumothorax

e 30-, 60-, and 90-day all-cause readmissions to the hospital in which the VATS lobectomy procedure was performed; $N$ with hospital-level follow-up data for at least 30 days = 620 Ethicon powered; 2279 Medtronic manual; for at least 60 days $=577$ Ethicon powered; 2210 Medtronic manual; for at least 90 days = 541 Ethicon powered; 2144 Medtronic manual

f Comprising chronic obstructive pulmonary disease and allied disorders, according to the ICD-9-CM/ICD-10-CM taxonomies; $n=323$ powered; 1020 manual 
Table 6 Multivariable-adjusted outcomes, Ethicon powered vascular stapler (PVS) and Medtronic manual post hoc subanalysis (years 2015 and 2016 only)

\begin{tabular}{|c|c|c|c|c|}
\hline \multirow[t]{2}{*}{$\overline{\text { Outcome }}$} & \multicolumn{2}{|c|}{ Adjusted outcomes ${ }^{a}$} & \multirow[t]{2}{*}{$P$} & \multirow{2}{*}{$\begin{array}{l}\text { \% difference } \\
(\text { powered - manual) }(\%)\end{array}$} \\
\hline & $\begin{array}{l}\text { PVS staplers, } \\
n=258\end{array}$ & $\begin{array}{l}\text { Medtronic manual } \\
\text { staplers, } n=793\end{array}$ & & \\
\hline Hospital LOS & 4.99 & 5.82 & 0.047 & -14.3 \\
\hline Total hospital costs & $\$ 23,219$ & $\$ 25,207$ & 0.242 & -7.9 \\
\hline Supply cost & $\$ 4693$ & $\$ 5728$ & 0.063 & -18.1 \\
\hline Room and board cost & $\$ 6714$ & $\$ 7955$ & 0.065 & -15.6 \\
\hline Operating room cost & $\$ 7294$ & $\$ 6752$ & 0.349 & 8.0 \\
\hline Discharge to non-home ${ }^{\mathrm{b}}$ & $9.7 \%$ & $7.3 \%$ & 0.497 & 32.5 \\
\hline Operating room time $\mathrm{c}^{\mathrm{c}}$ & 285 & 299 & 0.477 & -4.5 \\
\hline \multicolumn{5}{|l|}{ In-hospital complications } \\
\hline Bleeding/transfusion & $4.8 \%$ & $14.2 \%$ & 0.010 & -65.9 \\
\hline Transfusion & $3.0 \%$ & $9.4 \%$ & $0.023^{\mathrm{a}}$ & -68.4 \\
\hline APHA & $3.2 \%$ & $6.5 \%$ & 0.249 & -51.0 \\
\hline Air leak complications ${ }^{\mathrm{d}}$ & $20.9 \%$ & $26.2 \%$ & 0.255 & -20.4 \\
\hline Pneumonia & $0.9 \%$ & $2.7 \%$ & 0.202 & -68.0 \\
\hline Infection & $1.2 \%$ & $2.9 \%$ & 0.303 & -58.3 \\
\hline \multicolumn{5}{|l|}{ All-cause readmission ${ }^{\mathrm{e}}$} \\
\hline$\leq 30$ days & \multicolumn{4}{|c|}{ Model would not converge } \\
\hline$\leq 60$ days & $3.7 \%$ & $6.3 \%$ & 0.401 & -40.9 \\
\hline$\leq 90$ days & $3.7 \%$ & $8.5 \%$ & 0.210 & -56.2 \\
\hline
\end{tabular}

$A P H A$ acute posthemorrhagic anemia, LOS length of stay

${ }^{a}$ Recycled prediction method; The following outcome models had questionable fit due to estimated G matrix not positive definite: discharge to non-home; all-cause readmission at 30, 60, and 90 days; transfusion; air leak complications; pneumonia; infection

${ }^{\mathrm{b}}$ Discharge to a non-home setting (e.g., skilled nursing facility, intermediate care facility) vs. discharge to home with or without home healthcare

${ }^{c}$ In minutes; $n$ with operating room time data $=156$ PVS; 2190 Medtronic manual; operating room time values trimmed to fall between $30 \mathrm{~min}$ and $24 \mathrm{~h}$

d Composite of air leak and/or pneumothorax

e 30-, 60-, and 90-day all-cause readmissions to the hospital in which the VATS lobectomy procedure was performed; $N$ with hospital-level follow-up data for at least 30 days $=229$ PVS; 2279 Medtronic manual; for at least 60 days $=203$ PVS; 2210 Medtronic manual; for at least 90 days = 214 PVS; 2144 Medtronic manual

significant $(3.0 \%$ vs. $9.4 \%, P=0.023)$; however, the model had questionable statistical fit, likely due to the subanalysis' small sample size and low number of events, and therefore should be interpreted with caution. Differences between the study groups in all other outcomes were consistent with the directions of the primary analyses but not statistically significant.

In the subanalyses of patients with chronic obstructive pulmonary disease within the 
primary analysis stapler groups and the Ethicon powered and Medtronic manual stapler groups (Table 4, bottom panel; Table 5, bottom panel), the powered stapler group and Ethicon powered stapler group both had statistically significant lower adjusted rates of the composite hemostasis complication outcome (bleeding and/or transfusion) and transfusion compared with the manual stapler group and Medtronic manual stapler group.

Finally, in the propensity score matched sensitivity analysis for the primary analyses, all outcomes for which there was a statistically significant difference between the study groups remained statistically significant (Supplemental Table 3). After matching, all variables except for indicators for index hospitalization year of 2012 and 2013 had standardized differences less than 0.10 (Supplemental Tables 4-6) and thus all indicators for index hospitalization year were entered into the second-stage multivariable outcome models. The magnitude of differences tended to increase between the groups, and in one case (operating room time) results changed from statistically insignificant in the primary analysis to statistically significant in the propensity score matched sensitivity analysis, with the powered stapler group being associated with shorter adjusted operating room time compared with the manual stapler group.

\section{DISCUSSION}

To our knowledge, this study is the first realworld assessment of the association between the use of new generation powered and tissuespecific stapling technology on economic and clinical outcomes of VATS lobectomy procedures, as compared with the use of previous generations of manual staplers. As documented by various stakeholders worldwide-including researchers at the US Food and Drug Administration-real-world evidence (RWE) is gaining in popularity owing to its "potential for complementing the knowledge gained from traditional clinical trials, whose well-known limitations make it difficult to generalize findings to larger, more inclusive populations of patients, providers, and health care delivery systems or settings that reflect actual use in practice" [22].

In the primary and post hoc subanalyses, powered staplers (overall, Ethicon, and Ethicon PVS) were associated with $13.6-17.1 \%$ shorter length of stay as compared with manual staplers (overall, Medtronic). Although there are no prior published studies to which we can compare these specific results, the present study's average hospital LOS (4.7-5.7 days) are in the range of that reported in other US studies $[6,23,24]$. For example, among patients who underwent VATS lobectomy at the University of Kentucky Chandler Medical Center in years 2013-2014, the average hospital LOS was 5.5 days [6]. In a previous analysis of the Premier Healthcare Database examining patients who underwent VATS lobectomy in years 2007-2008, the average hospital LOS was 6.1 days [23]. Finally, in an analysis of the Nationwide Inpatient Sample database examining patients who underwent VATS lobectomy in years 2009-2012, the average hospital LOS was 6.2 days [24]. The slightly lower averages in the present study may be reflective of its relatively more contemporary time frame (2012-2016) coupled with a secular trend towards lower hospital LOS over time. Although we are unable to pinpoint the root cause of the shorter LOS associated with the powered stapler groups, one plausible explanation is their observed lower rates of hemostasis-related complications. Furthermore, as the Ethicon PVS stapler was designed to be specifically suitable for transecting pulmonary vessels, it is a notable finding that hemostasis-related complications were particularly low for this group. Given the relatively small sample size of the Ethicon PVS stapler group $(N=258)$, however, further research would be useful to corroborate these study findings.

Of particular significance are the findings of lower rates of transfusion associated with the use of Ethicon powered staplers. This finding was evident in the comparison between all Ethicon powered and Medtronic manual staplers, and directionally similar in the comparison between Ethicon PVS and Medtronic manual staplers but with questionable statistical fit for the underlying statistical model likely due 
to the small sample size and low number of events in that subanalysis. While this analysis is limited by the nature of the database in its ability to assess long-term outcomes, existing clinical literature suggests that perioperative blood transfusion during lung cancer resection is associated with a worse overall survival ( $R R$ $1.25, P<0.001)$, and worse recurrence-free survival (RR 1.42, $P<0.001$ )-which highlights the importance of adopting approaches and technologies associated with lesser associated risks of perioperative blood transfusion [25].

This analysis also demonstrated the greater likelihood of hemostasis-related complications and transfusion among patients with comorbid chronic obstructive pulmonary disease. This was also indicated in an earlier clinical study of lung cancer resection which suggested that proportions of patients with forced expiratory volume $(\mathrm{FEV})_{1}<60 \%$ needing perioperative blood transfusions were numerically twice that of patients with $\mathrm{FEV}_{1}>60 \%$ [19]. The association of Ethicon powered stapling devices with lower rates of reported transfusion suggests potential greater benefit among patients with progressive lung diseases.

Powered staplers (overall, Ethicon) were also associated with approximately 9\% lower total hospital costs (translating to differences of over $\$ 2000$ ) as compared with manual staplers (overall, Medtronic). Across the analyses, the subcategory of room and board costs appeared to be the main driver of the total hospital cost difference, a finding which is consistent with the aforementioned LOS differences. Notably, the subcategory of supply costs-in which the cost of staplers, reloads, and other surgical devices and products is captured-was not higher in the primary or subanalyses; thus, the use of powered staplers (and in the case of the Ethicon PVS stapler subanalysis, two powered staplers) did not appear to overwhelm any potential cost offsets which may be associated with these devices.

Powered staplers (overall, Ethicon, and Ethicon PVS) were consistently associated with lower rates of hemostasis-related complications. This finding, as well as the finding of lower total hospital costs, is consistent with a previous realworld study of patients who underwent laparoscopic Roux-en-Y gastric bypass or laparoscopic sleeve gastrectomy, wherein the use of powered staplers vs. manual staplers was associated with a lower rate of bleeding/transfusion and lower total hospital costs [14]. It should be noted that the rate of hemostasis-related complications in the present study is higher than reported in the clinical literature $[3,4,7]$. The higher rate of hemostasis-related complications in the present study is likely driven by differences in the method of ascertainment of these complications (coding vs. clinical observation). Indeed, as a result of the uncertainty of the sensitivity and specificity of the coding for hemostasis-related complications, this outcome was designated as exploratory and must be interpreted in the context of its associated limitations in real-world data sources such as miscoding or coding to drive increased reimbursement. However, we have no reason to assume that there would be systematic differential ICD coding errors across the study groups.

Two other prior studies provide results which are pertinent to the context of the present study's findings. First, a recent multicenter study conducted in China reported that complications were low during 94 VATS lobectomy procedures carried out specifically with Echelon Flex $^{\mathrm{TM}}$ powered ENDOPATH ${ }^{\circledR}$ staplers (Ethicon, Cincinnati, $\mathrm{OH}$ ) [26]. Additionally, in a questionnaire fielded to participating surgeons, 98.5\% reported that the articulation of the powered staplers made staple firing easier and 83.3\% reported it as essential, and the average stapler usability satisfaction score was 9 out of 10 [26]. Second, a recent preclinical study concluded that with its smaller profile, the four-row PVS stapler provided equivalent hemostasis to commercially available six-row staplers, while its more compact design provides easy, more precise access for vascular tissue stapling during minimally invasive procedures such as VATS lobectomy [27].

The observed associations between powered staplers and more desirable economic and clinical outcomes, specifically in control of hemostasis, may in part be attributed to a combination of stapler stability, articulation, control of tissue movement, and advanced 
staple reloads. These technological features may potentially allow for less damage to tissue and help with formation of a more integrated staple line. Furthermore, the PVS (narrow anvil, increased articulation, and smaller shaft diameter) was designed with the intention to optimize tissue-specific vessel firings, which in turn may confer better outcomes compared to staplers not specifically built for optimal tissue effects.

\section{LIMITATIONS}

This study was retrospective and non-randomized in design. Although multivariable regression analyses were used to control for multiple differences between the study groups, we cannot rule out the possibility of residual confounding. In particular, there were large differences in the regional distribution of hospitals, in the proportion of procedures with robotic assistance, and in the surgical volume of the hospitals, all of which are factors which may have had a strong influence on outcomes. However, in sensitivity analyses of the primary analyses, use of propensity score matching which balanced these factors across the cohorts did not substantially alter the study results. Thus, the propensity score matched sensitivity analysis demonstrates that the study results were robust to alternative statistical adjustment approaches, and that the large imbalance in hospital characteristics within the primary analysis did not lead to study results that were different relative to an approach in which those factors were balanced. We were unable to measure several potentially important patient-level variables within the study's data source which may represent unmeasured confounders, including smoking status, stage of cancer, prior operations, and prior radiation/chemotherapy. Additionally, factors such as surgeon and staff skill level, hospital-specific complication reduction efforts, and use of other medical devices were not measured and may have potentially confounded study results. Large databases that contain real-world administrative healthcare data are currently limited with respect to detailed information on medical devices. As a result of this data source limitation, we may not have captured all VATS procedures carried out with powered or manual staplers during the study period. Searches of non-standardized text fields in hospital billing data to identify use of staples may result in measurement error arising from incomplete capture of device descriptors and from inaccurate assignment of descriptors for devices within hospital billing systems.

\section{CONCLUSION}

In this analysis of real-world data from VATS lobectomy procedures, powered staplers were associated with significant benefits with respect to selected types of hospital resource use, costs, and clinical outcomes when compared with manual staplers.

\section{ACKNOWLEDGEMENTS}

Funding. Sponsorship for this study, the article processing charges, and Open Access fee was funded by Johnson \& Johnson (Ethicon, Somerville, NJ).

Medical Writing and/or Editorial Assistance. We would like to acknowledge Jay Lin, $\mathrm{PhD}$ and Melissa Lingohr-Smith, PhD from Novosys Health in the editorial support and review of this manuscript.

Authorship. All named authors meet the International Committee of Medical Journal Editors (ICMJE) criteria for authorship for this manuscript, take responsibility for the integrity of the work as a whole, and have given final approval to the version to be published. All authors had full access to all of the data in this study and take complete responsibility for the integrity of the data and accuracy of the data analysis.

Thanking Participants. We thank the participants of the study, whose identities are anonymous as a result of the retrospective, deidentified nature of the study. 
Disclosures. Daniel L. Miller: Scientific advisory board, Ethicon; Speakers Bureau, Medtronic; Scientific advisory board, Pacira; Speakers Bureau, Acute Innovations. Stephen S. Johnston is an employee of Johnson \& Johnson and a Johnson \& Johnson stockholder. Sushama Ramisetti is an employee of Johnson \& Johnson. Sashi Yadalam is an employee of Johnson \& Johnson. Edmund S. Kassis is an employee of Johnson \& Johnson and a Johnson \& Johnson stockholder. Sanjoy Roy is an employee of Johnson \& Johnson and a Johnson \& Johnson stockholder.

Compliance with Ethics Guidelines. This study was conducted per a prespecified protocol which was approved through scientific governance. As a result of the retrospective, observational nature of the study, it was not a registered trial. The study was planned and conducted in a manner consistent with the International Society of Pharmacoepidemiology principles of the Good Research for Comparative Effectiveness guidance and the PICO Model for Clinical Questions. The Premier Healthcare Database consists of de-identified healthcare records. In the USA, retrospective analyses of the Premier Healthcare Database data are considered exempt from institutional review board (IRB) oversight as dictated by Title 45 Code of Federal Regulations, Part 46 of the USA, specifically 45 CFR 46.101(b)(4) (http://www. hhs.gov/ohrp/humansubjects/guidance/45cfr46. html). In addition, in accordance with the HIPAA Privacy Rule, disclosed data from the Premier Healthcare Database are considered de-identified per 45 CFR 164.506(d)(2)(ii)(B) through the "Expert Determination" method (http://www. hhs.gov/hipaa/for-professionals/privacy/specialtopics/de-identification/). Throughout this research project, the study data remained deidentified and stored on encrypted, passwordprotected servers to protect patient confidentiality. This article does not contain any studies with human participants or animals performed by any of the authors.

Data Availability. The datasets generated and/or analyzed during the current study are not publicly available because of contractual arrangements with the Premier Inc. The Premier
Healthcare Database may be obtained directly from Premier Inc.

Open Access. This article is distributed under the terms of the Creative Commons Attribution-NonCommercial 4.0 International License (http://creativecommons.org/licenses/ by-nc/4.0/), which permits any noncommercial use, distribution, and reproduction in any medium, provided you give appropriate credit to the original author(s) and the source, provide a link to the Creative Commons license, and indicate if changes were made.

\section{REFERENCES}

1. Roviaro GC, Varoli F, Vergani C, Maciocco M. State of the art in thoracoscopic surgery: a personal experience of 2000 videothoracic procedures and an overview of the literature. Surg Endosc. 2002;16:881-92.

2. Solaini L, Prusciano F, Bagioni P, et al. Video-assisted thoracic surgery (VATS) of the lung: analysis of intraoperative and postoperative complications over 15 years and review of the literature. Surg Endosc. 2008;22:298-310.

3. Imperatori A, Rotola N, Gatti M, et al. Peri-operative complications of video-assisted thoracic surgery (VATS). Int J Surg. 2008;6(Suppl 1):S78-81.

4. Laursen LO, Petersen RH, Hansen HJ, et al. Videoassisted thoracic surgery lobectomy for lung cancer is associated with a lower 30-day morbidity compared with lobectomy by thoracotomy. Eur J Cardiothorac Surg. 2016;49:870-5.

5. Bendixen $\mathrm{M}$, Jorgensen $\mathrm{OD}$, Kronborg $\mathrm{C}$, et al. Postoperative pain and quality of life after lobectomy via video-assisted thoracic surgery or anterolateral thoracotomy for early stage lung cancer: a randomised controlled trial. Lancet Oncol. 2016;17:836-44.

6. Rodgers-Fishl PM, Martin JT, Saha SP. Video-assisted thoracic versus open lobectomy: costs and outcomes. South Med J. 2017;110:229-33.

7. Mafe JJ, Planelles B, Asensio S, et al. Cost and effectiveness of lung lobectomy by video-assisted thoracic surgery for lung cancer. J Thorac Dis. 2017;9:2534-43.

8. Business Wire. Covidien receives FDA 510(k) clearance for the iDriveTM powered stapling system. 
Business Wire, San Francisco. 2 Dec 2010. http:// www.businesswire.com/news/home/201012020051 77/en/CovidienMedtronic-Receives-FDA-510-Clear ance-iDrive \%E2\%84\%A2-Powered. Accessed 15 Nov 2017.

9. Echelon Flex Endopath Staplers. Ethicon. Cincinnati. http://www.ethicon.com/healthcare-profess ionals/products/staplers/endocutters/echelon-flexendopath\#!description-and-specs. Accessed 15 Nov 2017.

10. PR Newswire. Ethicon endo-surgery introduces first powered endocutter with enhanced system-wide compression and stability, allowing surgeons greater control in laparoscopic surgery. Cision, Chicago. 27 Sept 2011. http://www.prnewswire. com/news-releases/ethicon-endo-surgery-introducesfirst-poweredendocutter-with-enhanced-system-widecompression-and-stabilityallowing-surgeons-greatercontrol-in-laparoscopic-surgery-130591488.html. Accessed 15 Nov 2017.

11. iDrive Ultra Powered Stapling System. Medtronic. Minneapolis. http://www.medtronic.com/content/ dam/covidien/library/us/en/product/surgical-stapli ng/idrive-ultra-powered-stapling-system-guide-forsurgeons.pdf. Accessed 15 Nov 2017.

12. Echelon Flex GST System. Ethicon. Cincinnati. http://www.ethicon.com/healthcare-professionals/ products/staplers/endocutters/echelon-flex-gst-system. Accessed 15 Nov 2017.

13. Echelon Flex Powered Vascular Stapler. Ethicon. Cincinnati. http://www.ethicon.com/healthcareprofessionals/products/staplers/endocutters/echelonflex-powered-vascular-stapler\#!science-and-technol ogy. Accessed 15 Nov 2017.

14. Roy S, Yoo A, Yadalam S, et al. Comparison of economic and clinical outcomes between patients undergoing laparoscopic bariatric surgery with powered versus manual laparoscopic surgical staplers. J Med Econ. 2017;20:423-33.

15. Quan H, Sundararajan V, Halfon P, et al. Coding algorithms for defining comorbidities in ICD-9-CM and ICD-10 administrative data. Med Care. 2005;43:1130-9.

16. Manning WG, Mullahy J. Estimating log models: to transform or not to transform? J Health Econ. 2001;20:461-94.
17. Park RE. Estimation with heteroscedastic error terms. Econometrica. 1996;34:888.

18. Muller CJ, MacLehose RF. Estimating predicted probabilities from logistic regression: different methods correspond to different target populations. Int J Epidemiol. 2014;43:962-70.

19. Licker MJ, Widikker I, Robert J, et al. Operative mortality and respiratory complications after lung resection for cancer: impact of chronic obstructive pulmonary disease and time trends. Ann Thorac Surg. 2006;81:1830-7.

20. Grace principles. Good ReseArch for Comparative Effectiveness. Cambridge. 10 Apr 2010. https:// www.pharmacoepi.org/pub/1c29f69f-2354-d7145100-1ef2b0e9abd9. Accessed 15 Nov 2017.

21. Sackett D, Richardson WS, Rosenburg W, Haynes RB. How to practice and teach evidence based medicine. 2nd ed. London: Churchill Livingstone; 1997.

22. Sharman RE, Anderson SA, Dal Pan GJ, et al. Realworld evidence-what is it and what can it tell us? N Engl J Med. 2016;375:2293-7.

23. Swanson SJ, Meyers BF, Gunnarsson CL, et al. Video-assisted thoracic lobectomy is less costly and morbid than open lobectomy: a retrospective multiinstitutional database analysis. Ann Thorac Surg. 2012;93:1027-32.

24. Desai H, Natt B, Kim S, Bime C. Decreased in-hospital mortality after lobectomy using video-assisted thoracic surgery compared with open thoracotomy. Ann Am Thorac Soc. 2017;14:262-6.

25. Luan H, Ye F, Wu L, Zhou Y, Jiang J. Perioperative blood transfusion adversely affects prognosis after resection of lung cancer: a systematic review and a meta-analysis. BMC Surg. 2014;14:34. https://doi. org/10.1186/1471-2482-14-34.

26. Qiu B, Yan W, Chen K. A multi-center evaluation of a powered surgical stapler in video-assisted thoracic lung resection procedures in China. J Thorac Dis. 2016;8:1007-13.

27. Ng CSH, Pickens A, Siegel JM, Clymer JW, Cummings JF. A novel narrow profile articulating powered vascular stapler provides superior access and haemostasis equivalent to conventional devices. Eur J Cardiothorac Surg. 2016;Suppl 1:i73-8. 